\title{
Discussion on Approaches to Cultivate Personality Charm of TV Programme Hosts
}

\author{
Qiaoling Dai ${ }^{1}$, Shimin Yang $^{2}$ \\ ${ }^{1}$ Department of Theatricals, Jiangxi Vocational Academy of Art, Nanchang, Jiangxi, 330024 \\ ${ }^{2}$ Science and Technology College, Jiangxi Normal University, Nanchang, Jiangxi, 330024
}

Keywords: TV programme, Host, Personality charm

\begin{abstract}
Personal charm is a kind of force which can generate strong attraction. Hosts' personality charm is a decisive factor of audience rating of TV programmes and also the soul of a programme. This paper starts from the composition of personality charm of TV programmes, analyzes development direction of each component in detail and studies the cultivation approaches, in the hope of really giving play to advantage of hosts' personality charm, promoting further development of TV programmes and forming correct guidance of social consciousness.
\end{abstract}

\section{Introduction}

Different people have diverse personality characteristics, and their personality charm also presents large differences. Personality charm as an important index to overall evaluate hosts in current society is a significant driving factor for TV media developing to host media. Personality charm of TV programme hosts is external expression form of their comprehensive quality and reflects their humanistic connotation, aesthetic standards, artistic culture and wisdom. In recent years, as social economy further develops, the reform and opening continuously deepens. With further development of science and technology, competitions among mainstream media become increasingly fierce. In order to gain advantages in market competitions and meet needs of social public for media culture, each TV station launches various kinds of programmes and corresponding hosts, and provides richer selections and more superior services foe social public. It's worth noting that among these programmes, the hosts with high audience rating which are welcomed by social public generally have high personality charm. It thus can be seen that hosts' personality charm imposes important influence on TV programmes.

\section{Composition of personality charm of TV programme hosts}

\section{External image of programme hosts}

The host as image representative of TV programmes will generate important influence on image of TV programmes through his/her any action or language. In general, hosts with natural and decent behaviors can be more easily widely accepted by programme audiences, while the harmony of hosts' personality charm and programme nature should be valued by programme recording. Thus, during arranging programmes, hosts' external image and aesthetic demand of audiences should be mainly valued. To position image for hosts, it is required to be natural and real to arouse favorable impression of social public and avoid occurrence of vulgar phenomena.

Meanwhile, as broadcasting and TV further develop and become mature, hosts' external image is endowed with new connotation by the ear. It is no longer pure shallow image which represents hosts' appearance and clothes. It to some extent represents outward manifestation of hosts' comprehensive quality. Hosts with sufficient ability are further welcomed by social public. Cui Yongyuan, Bai Yansong, Li Yong and Bi Fujian are the typical representatives. They do not belong to handsome men within social public aesthetic tendency, but gradually become popular due to their wisdom, culture and humorous language. They represent mainstream development trend of charm cultivation of hosts. 


\section{Inherent temperament of programme hosts}

Inherent temperament of programme hosts depends on their congenital characters and is influenced by knowledge structure. The difference in inherent temperament of programme hosts drives programmes to show corresponding personality characteristics. The hosts with excellent inherent temperament can not just make programmes rich and colorful, but also provide certain guarantee for programme audience rating. Seeing from the comprehensive level, inherent temperament of programme hosts is essential attribute of their personality charm. One's living environment, life experience and education degree will influence inherent temperament. To make hosts show excellent quality and demeanor in programme recording process, it is required to start from hosts' experience and education degree, make them firmly grasp attention of programme audiences while completing their own training, boost audience rating through hosts' personality charm and promote further development of TV programmes.

\section{Hosts' language style with distinct personality}

Language as an affective communication tool of TV programme hosts is a display platform of hosts' personality charm. To be more specific, language style of TV programme hosts is mainly expressed in two aspects: voiced language and silent language (posture). In a bid to make TV programme hosts convey the most sincere emotion to audiences through rhythmic language, it is required to start from external image of language and apply personification, exaggeration, metaphor and metaphor to make hosts' charm and talents shown in programmes so as to attract audiences' attention to the largest extent and boost programme audience rating. In terms of specific operation, hosts should specify substantive characteristics of programmes, rationally plan and organize language. For example, hosts of law programmes should attach importance to language seriousness and logic. Hosts of entertainment programmes should focus on language vitality and interestingness. Hosts of life programmes should lay emphasis on language kindness and randomness. Hence, to ensure programme recording effect and improve programme level, hosts should train language style with bright personality characteristics and create unique programme image.

\section{Character training of programme hosts}

It can be seen from current development status of TV programmes that some TV programme hosts convey dense exploration spirit in TV programmes due to influence of social advanced media culture so that TV programmes are flexible, varied, rich and colorful and also reflect hosts' unique personality charm features. Meanwhile, some TV programme hosts present very keen political sensitivity due to the influence of socialist spiritual civilization. Besides, it is reflected in programmes and reflects abomination of dark and ugly phenomena and praise of the good and the beautiful. This plays a correct guiding role for thought of social public. These TV programmes also gain favorable development, and the audience rating keeps a high level. It thus can be seen that excellent morality has very important effect on hosts' personality charm growth. Therefore, TV programme hosts should positively cultivate their morality, establish correct social consciousness and actively make the programme a significant symbol of the whole city and even the state.

\section{Aesthetic tendency of programme hosts}

Aesthetic tendency of TV programme hosts impose important influence on whole programme quality and style formation. Certain thought construction and aesthetic thinking jointly maintain the core of the whole programme. Even for some political comment programmes with epic deductive nature, although these programmes aim to provide certain standards and ideological restriction for constructing mainstream social ideology and social ideology, hosts also may provide more superior services for social public through expressing their aesthetic tendency and venting strong thoughts and emotions in order to cater to aesthetic needs the audiences. In numerous geography, life, history and culture TV programmes, hosts' aesthetic tendency expression will be better exerted. In particular, aesthetic expression with personality characteristics can more easily form their unique style in programme recording process so as to attract audiences and create more social and economic benefits for programmes. 


\section{Approaches to cultivate personality charm of TV programme hosts}

TV is a popular entertainment tool with the most extensive audiences. Hence, for advancement and healthy development of social mainstream ideology, TV programmes should be devoted to providing abundant and wonderful news entertainment programmes with high taste, promote national quality in knowledge information communication and further meet modern needs of the public in spiritual culture construction. This paper proposes cultivation suggestions for personality charm from the following aspects in allusion to components of personality charm of TV programme hosts, hopes to boost TV programme level and promote development of TV media.

\section{To further boost political theory quality of hosts}

TV media is the most influential media means in current society and represents information transmission channels of the party and state to some extent. TV programme hosts as main carriers of information communication have significant influence on depth and quality of information communication. Since Chinese government issued professional ethics of TV programme hosts in the form of documents in 2004, all provinces and cities have started to pay attention to professional ethics construction of hosts, and striven to make hosts bear the lofty mission of carrying forward Chinese advanced culture, inheriting great national spirit, maintaining national and social benefit and driving economic development so as to further enhance socialism spiritual civilization construction. So, current TV programme hosts must own high political theory quality and level of professional ethics, show such quality in language and behaviors and generate positive effects on social public.

\section{To further strengthen professional quality construction of hosts}

In current social environment with media social environment, due to the influence of hosts on programme audiences, TV programme image prepared and organized by hosts are called “hosts' own programmes" vividly. Thus, hosts should not merely complete basic recording work in programme recording process, but also attach importance to possible effects of programmes on social policies, economy and culture. Therefore, hosts must enhance their own professional quality construction and better complete missions of programmes. In China, hosts' professional quality is often divided into the following aspects: political sensitivity quality, professional knowledge quality, humanistic quality, physiological and psychological quality etc. These play a decisive role for hosts' career success. Hence, in future career development construction, hosts should firstly correct programme hosting attitude and guide positive social opinions through harmonious language, intonation, action and expression. Besides, hosts should actively train their ability to deal with emergencies and keep smooth connection of programmes when inter-cut and cutting phenomena appear. Secondly, to boost programme quality, hosts should read extensively, actively improve their knowledge reserve, perfectly combine the knowledge and programmes in continuous exploration and practice process and cultivate humanistic thought connotation of social public while guaranteeing programme quality.

\section{To explore hosting style with bright personality}

Excellent TV programme hosts become a programme brand and are widely welcomed by social public. This is mainly because their unique personality charm can be accepted and favored by social public. Hosting style with bright personality is not just reflected in language style and external image peculiarity, but also covers experience accumulation and emotional experience gain in growing process. Such unique external image, language style and internal ideological implication jointly form individual hosting style of hosts. Even hosts once became the spokespersons of programme nature. Only when hosts own rich emotional experience, profound humanistic knowledge and super-strong flexibility can they gradually form their own style in programme hosting process so as to boost programme audience rating through displaying personality charm.

\section{To enhance field control ability training with personality characteristics}

Reinforcement ways of field control ability include two: firstly, reinforce field control ability with field control skills. During implementation of this way, hosts should own the ability to transform 
interview modes according to the object of interview. For example, during having an interview with honored guests in the industry, hosts should know these honored guests are often very familiar with hosts' intension and skills. They have entered interview theme when hosts do not end the statement. So, hosts should prepare well the interview outline and theme in advance and drive their mood flexibly in the interview process so as to control the field well and improve programme effects. For the guests with low enthusiasm for the programme, hosts should firstly actively mobilize their work enthusiasm and overawe others by rich experience and knowledge. In this way, guests can relax and actively put themselves in programme recording so as to complete programme recording in an efficient manner. Secondly, hoots may enhance field control ability through humorous enthusiasm. Humorous hosting style can activate the atmosphere of recording field, eliminate guests' tension, facilitate affective interaction between guests and hosts, male programme depth and width increase and provide more superior services for social public.

\section{Conclusions}

As broadcasting and TV programmes further develop, hosts’ personality charm plays a decision role for future development of programmes. Based on analyzing personality charm components of TV programme hosts in detail, this paper deeply discusses the ways to improve personality charm and hopes to provide certain help for continuous and healthy development of Chinese TV programmes through cultivating hosts’ personality charm.

\section{References}

[1] Ma Tong, Du Xiao, On approaches to cultivate personality charm of TV programme hosts. Chinese \& Foreign Entrepreneurs, 2013(7).

[2] He Jia, More and more attention to language individuality charm in current TV industry. Business Conditions, 2013(31)

[3] Pei Lingling, How to manifest personality charm of TV talk show hosts. The Spread of Science and Technology Magazine, 2013(22)

[4] Sheng Lijun, Du Xiao, Expression forms of personality charm of TV programme hosts in recent years. Chinese \& Foreign Entrepreneurs, 2013(5)

[5] Zhang Wenjing, Construction of personality charm of reporter-based TV programme hosts. Youthful Days, 2012(20)

[6] LI Qian, Analysis of personality charm of TV programme hosts. Shenzhou (mid-month periodical), 2014(3)

[7] Liu Guohua, On TV programme hosting art from perspective of personality charm. SAN WEN BAI JIA XUE SHU BAI JIA, 2012,02(1)

[8] Lian Jing, On personality charm shaping of sports programme hosts - case study of Comments on Sports. Journal of Harbin Institute of Vocational Technology, 2014(2)

[9] Huang Gang, Analysis of shaping of hosts' personality charm on TV programme quality. News Knowledge, 2012(4) 